
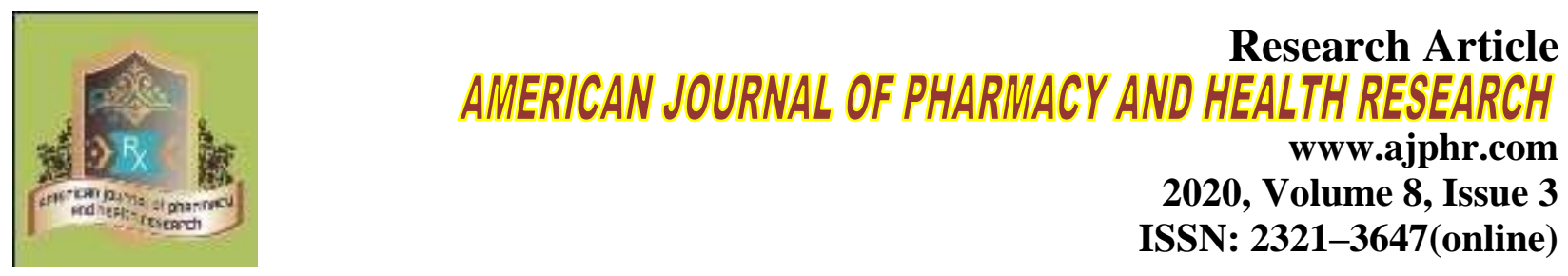

\title{
Novel Approach for Design and Characterization Of Muco Adhesive Buccal Ganciclovir In Situ Gel
}

\author{
CH.N.V.S.Masthan Rao* R.B.Desireddy, D.Vasavilatha, G.L.S.Mounika, G.Ramya \\ Sadhana , K.L.T.Sowjanya \\ Nalanda Institute of Pharmaceutical Sciences, Kantepudi, Guntur
}

\begin{abstract}
The present study was design to prepare periodontal gel of Ganciclovir for the treatment of inflammation condition. In this study, it was found that as the phospholipid concentration was increased, it resulted in corresponding increase in the entrapment efficiency of reconstituted liposomes. In conclusion, a sustained delivery of Ganciclovir can be achieved by proliposomal drug delivery system. Phospholipids, being the major component of liposomal system, can easily get integrated with the skin lipids and maintain the desired hydration conditions to improve drug permeation. Fusion of lipid vesicles with skin contributed to the permeation enhancement effect. In -vitro studies concluded that enhance skin permeation and retention of Ganciclovir was observed and was due to liposolubulized state of drugs with in proliposomes which helped to produce the depot effect. The prepared proliposomes are in corporated in to gel. The data show that liposomal systems can make the drug molecule more accessible with in skin layers. This conclusion compares favourably with early study showed that drug associated with liposomes bilayer bounds and better routed into skin. Other studies have shown that liposomal ambiaence may help modify the permeability characteristics of the stratum corneum, and the system keep the drug molecules with in skin layers so that sustain release of drug can be achieved. The results advocate the extension of this work on the preliminary clinical trails and commercialization of proliposomal gel formulation of anti viral drugs for effective topical pharmacotherapy in treatment of virus.
\end{abstract}

Keywords: Ganciclovir, Gellan gum, Mucoadhesive in situ gel

*Corresponding Author Email: anammahime@gmail.com; diadora832003@yahoo.fr

Received 10 February 2020, Accepted 22 February 2020 


\section{INTRODUCTION}

The unique environment of the oral cavity offers its potential as a site for drug delivery. Because of the rich blood supply and direct access to systemic circulation, the oral mucosal route is situated for drug, which are susceptible to acid hydrolysis in the stomach or which are extensively metabolized in the liver (first pass effect). The total area of the oral cavity is about $100 \mathrm{~cm}^{3}$. Out of this about one third is the buccal surface, which is lined with an epithelium of above $0.5 \mathrm{~mm}$ thickness. The oral mucosal surface is continuously washed by the saliva (daily turn out is about 0.5 to 2 liters).

\section{Delivery through Buccal Mucosa:}

Administration of a drug via the buccal mucosa (the lining of the cheek) to the systemic circulation is defined as buccal delivery. Despite, the buccal mucosa is significantly less permeable than the sublingual mucosa and usually not able to provide rapid drug absorption or good bio availability, it is relatively more permeable than sublingual floor makes it more desirable site for sustained drug delivery. Apart from avoiding enzymatic degradation and first pass metabolism, the non acidic conditions and lipophillic nature of the buccal tissue provide potential and promises for successful delivery of peptide and proteins.

\section{The various strategies Employed for Buccal Delivery}

*Bio adhesive buccal Gels

\section{Bioadhesivebuccal Gels:}

Viscous adhesive gels have been designed for local therapy using polyacrylic acid and polymethacrylate as gel forming polymers. Gels are reported to prolong residence time on the oral mucosa to a significant level. This not only improves absorption but also allows for sustained release of the active principle.

Include solutions, suspensions, gels, micro particles, suppositories, creams, foams and tablets. And all have relatively short contact time. Robinson et al reported on a system of treatment using a gel containing the mucoadhesive epolycarbophil that remained on vaginal tissue for 3-4 days and hence served as a platform for delivery of drug such as progesterone.

\section{MUCOADHESIVE DRUG DELIVERY}

The potential route of buccal mucosal route of drug administration was first recognized by Walton and others reported in detail on the kinetics of buccal mucosal absorption.

Mucoadhesion, or the attachment of a natural or synthetic polymer to a biological substrate, is a practical method of drug immobilization or localization and an important new aspect of 
controlled drug delivery. The unique environment of the oral (buccal) cavity offers its potential as a site for drug delivery. Because of the rich blood supply and direct access to systemic circulation. The Buccal route is suitable for drugs, which are susceptible to acid hydrolysis.

\section{LIPOSOMES}

- Liposomes are concentric bilayered vesicles in which an aqueous volume is entirely enclosed by a membranous lipid bilayer mainly composed of natural or synthetic phospholipids. The water soluble compounds/drugs are present in aqueous compartments while lipid soluble compounds/drugs and amphiphilic compounds or drugs insert themselves in phospholipid bilayers.

- The liposomes containing drugs can be administrated by many routes (intravenous, oral inhalation, local application, ocular) and these can be used for the treatment of various diseases.

- For drug delivery applications liposomes are usually unilamellar and range in diameter from about $50-150 \mathrm{~nm}$.

- Depending on the lipid composition, methods of preparations and the nature of the encapsulated agents, many types of liposomal products can be formulated.

\section{Advantages of liposomes}

- Provides selective targeting to tumour tissues.

- Increased efficiency and therapeutic index.

\section{Disadvantages of liposomes}

- Liposomes are bio compatible.

- Liposome-incorporated pharmaceuticals are protected from the inactivating effect of external conditions, yet do not cause undesirable side reactions.

\section{Preparation methods:}

The formulation of an appropriate liposomal system as a carrier for a given drug is dependent on the type of the lipid used and the method of preparation.

\section{Ethanol Injection Method:}

A lipid solution of ethanol is rapidly injected to a vast excess of buffer. The MLVs are immediately formed. The drawbacks of the method are that the population is heterogeneous (30$110 \mathrm{~nm}$ ), liposomes are very dilute, it is difficult to remove all ethanol.

\section{Ether Infusion Method:}


A solution of lipids dissolved in diethyl ether or ether/methanol mixture is slowly injected to an aqueous solution of the material to be encapsulated at $55-65^{\circ} \mathrm{C}$ or under reduced pressure. The subsequent removal of ether under vacuum leads to the formation of liposomes. The main drawbacks of the method are that the population is heterogeneous $(70-190 \mathrm{~nm})$ and the exposure of compounds to be encapsulated to organic solvents or high temperature.

\section{Reverse Phase Evaporation Method:}

First water in oil emulsion is formed by brief sonication of a two phase system containing phospholipids in organic solvent (diethylether or isopropylether or mixture of isopropyl ether and chloroform) and aqueous buffer. The organic solvents are removed under reduced pressure, resulting in the formation of a viscous gel. The liposomes are formed when residual solvent is removed by continued rotary evaporation under reduced pressure.

\section{Lipid Hydration Method:}

This is the most widely used method for the preparation of MLV. The method involves drying a solution of lipids so that a thin film is formed at the bottom of round bottom flask and then hydrating the film by adding aqueous buffer and vortexing the dispersion for some time.

\section{Freeze-Thaw Method:}

SUVs are rapidly frozen and followed by slow thawing. The brief sonication disperses aggregated materials to LUV. The formation of unilamellar vesicles is due to the fusion of SUV during the processes of freezing and or thawing.

\section{Solvent Spherule Method:}

The process involved dispersing in aqueous solution the small spherules of volatile hydrophobic solvent in which lipids had been dissolved. MLVs were formed when controlled evaporation of organic solvent occurred in a water bath

\section{Applications of liposomes}

(1) Liposomes act as carriers for drug both in vitro and in vivo.

(2) In targeted drug delivery system, liposomes are used as carriers of anticancer drug such as methotrexate, actinomycin- D etc.

(3) Liposomes encapsulation has been used as a step in enzyme purification.

\section{DRUG PROFILE}

$\begin{array}{lll}\text { Drug name } & : \text { Ganciclovir } \\ \text { IUPAC name } & : 2 \text {-amino-9- }\{[(1,3 \text {-dihydroxypropan-2-yl)oxy }] \text { methyl }\}-6,9 \text {-dihydro-- purin - }\end{array}$ 6- one. 
Synonyms :Ganciclovir, GA2, 9-[(1,3-dihydroxy-2-propoxy)methyl]guanine,

Solubility : Soluble in $0.1 \mathrm{M} \mathrm{HCl}(10 \mathrm{mg} / \mathrm{ml})$, DMSO $(5 \mathrm{mg} / \mathrm{ml})$, water $(2$

$\mathrm{mg} / \mathrm{ml})$, hot methanol, and ethanol $(<1 \mathrm{mg} / \mathrm{ml})$.

Melting point $: 250^{\circ} \mathrm{C}$

Structure :

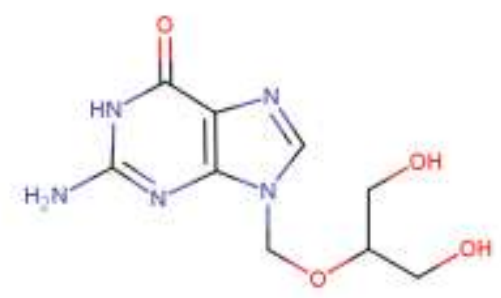

Molecular formula : C9H13N5O4

Molecular weight :Average: 255.2306 Monoisotopic: $255.096753929 \mathrm{~g} / \mathrm{mol}$.

Bioavailability : 5\%(oral)

Half-life $\quad: 2.5$ to 3.6 hours

Protein binding : 1 to $2 \%$

Dosage forms : injection, gel, powder, capsule.

Dose $\quad: 250,500 \mathrm{mg} .500 \mathrm{mg} / 10 \mathrm{ml}$.

Category :Antiviral Agents

\section{MATERIALS AND METHOD}

The following materials are used for project work

\begin{tabular}{lll}
\hline S.No. & Materials & Source \\
\hline 1 & Ganciclovir & A gift sample from Dr. Reddy's laboratories, Hyderabad. AP. \\
2 & Mannitol & SD Fine-Chem. Pvt., Mumbai, India. \\
3 & Cholesterol & SD Fine-Chem. Pvt., Mumbai, India. \\
4 & Phosphotidyl choline & Dr. Reddy's laboratories, Hyderabad, AP. \\
5 & Chloroform & Merk laboratories, Mumbai \\
6 & Methanol & Merk laboratories, Mumbai \\
7 & Gellan gum & Yaro chemicals, Mumbai \\
8 & Triethanolamine & Fisher scientific, Mumbai \\
\hline
\end{tabular}

\section{List of chemicals used with supplier}

Instruments Used:

\begin{tabular}{llll}
\hline S.No. & Instrument name & Manufacturer & Model \\
\hline 1 & UV spectrophotometer & Lab India & 32000 \\
2 & Rota evaporator & Helidopath & Sonics-569-00050-00-0 \\
4 & Optical microscope & Metzer & 5000DTM \\
5 & Viscometer & Brook field & LVDV II pro+ \\
6 & Magnetic stirrer & Remi & 1ML \\
\hline
\end{tabular}




\begin{tabular}{llll}
\hline 7 & Zeta sizer & Malvern & \\
8 & Hot air oven & Navtug & D5247 \\
9 & Weighing balance & Shimadzu & Apx224 \\
10 & Water purifier & Labindia & SGLABOSTAR3TWF \\
11 & Scanning electron microscope & & \\
12 & $\mathrm{P}^{\mathrm{H}}$ meter & Lab India & SAB 5000 \\
13 & FTIR & Brucker & Alpha-T \\
14 & KBR Pellet Press & Horizon & WC-56 \\
15 & Ultra Sonicator & Citizen & CD4520 \\
16 & Cooling centrifuge & REMI & TR-01 \\
17 & Homogenizer & REMI & RQT-124A \\
\hline
\end{tabular}

\section{List of Equipments used}

METHODOLOGY:

\section{Preformulation studies:}

Preformulation testing is an investigation of physical and chemical properties of a drug substance alone and when combined with excipients. It is the first step in the rational development of dosage forms.

\section{Objective}

The overall objective of preformulation testing is to generate information useful to the formulation in developing stable and bioavailable dosage forms.

\section{Scope}

The use of preformulation parameters maximizes the chances in formulating an acceptable, safe, efficacious and stable product.

\section{Determination of absorption maxima:}

Absorption maxima is the wavelength at which maximum absorption takes place. For accurate analytical work, it is important to determine the absorption maxima of the substance under study.

\section{Procedure:}

For the preparation of calibration curve stock solution was prepared by dissolving $100 \mathrm{mg}$ of accurately weighed drug in $100 \mathrm{ml}$ of methanol $(1 \mathrm{mg} / \mathrm{ml})$. Further $1 \mathrm{ml}$ of the stock solution was pipette out into a $100 \mathrm{ml}$ volumetric flask and volume was made up with phosphate buffer (7.4 $\mathrm{PH})$. From this stock solution pipette out $1 \mathrm{ml}$ and dilute to $10 \mathrm{ml}$ with phosphate buffer and subject for UV scanning in the range of 200-400 nm using double beam UV spectrophotometer. The absorption maxima was obtained at $270 \mathrm{~nm}$ with a characteristic peak.

\section{Drug-excipients interaction studies by FTIR :}

The compatibility between pure drug and mannitol, phosphatidyl choline, cholesterol were detected by FTIR (Bruker Alpha- T) spectra. The potassium bromide pellets were prepared on 
KBR press (Horizon WC-56). To prepare the pellets the solid powder sample were ground together in a mortar with 100 times quantity of KBR. The finely grounded powder introduced into a stainless steel die. The powder was pressed in the die between polished steel anvils at a pressure of about 10t/in' . The spectra's were recorded over the wave number of 4000 to $600 \mathrm{~cm}^{-1}$ Preparation of Ganciclovir loaded proliposomes:

Proliposomes could be prepared by many methods including

- Film deposition on carrier method

- Crystal film method

- Fluidized bed method

- Powder bed grinding method

- Freeze drying method

- Spray drying method

Based on the laboratory conditions film-deposition on the carrier method was chosen to prepare Ganciclovir proliposomes.

Variables for optimization of proliposomes:

- Mannitol

- Phosphatidyl choline

- Cholesterol

\section{RESULTS AND DISCUSSION}

Preformulation studies:

Melting point determination: Melting point of Ganciclovir was determine by capillary method. Melting point of Ganciclovir was found to be in the range of $152-155^{\circ} \mathrm{C}$ which compiles the standards thus indicating that purity of the drug sample.

Construction of calibration curve using phosphate buffer $\left(\mathbf{P}^{\mathrm{H}} \mathbf{7 . 4}\right)$ 


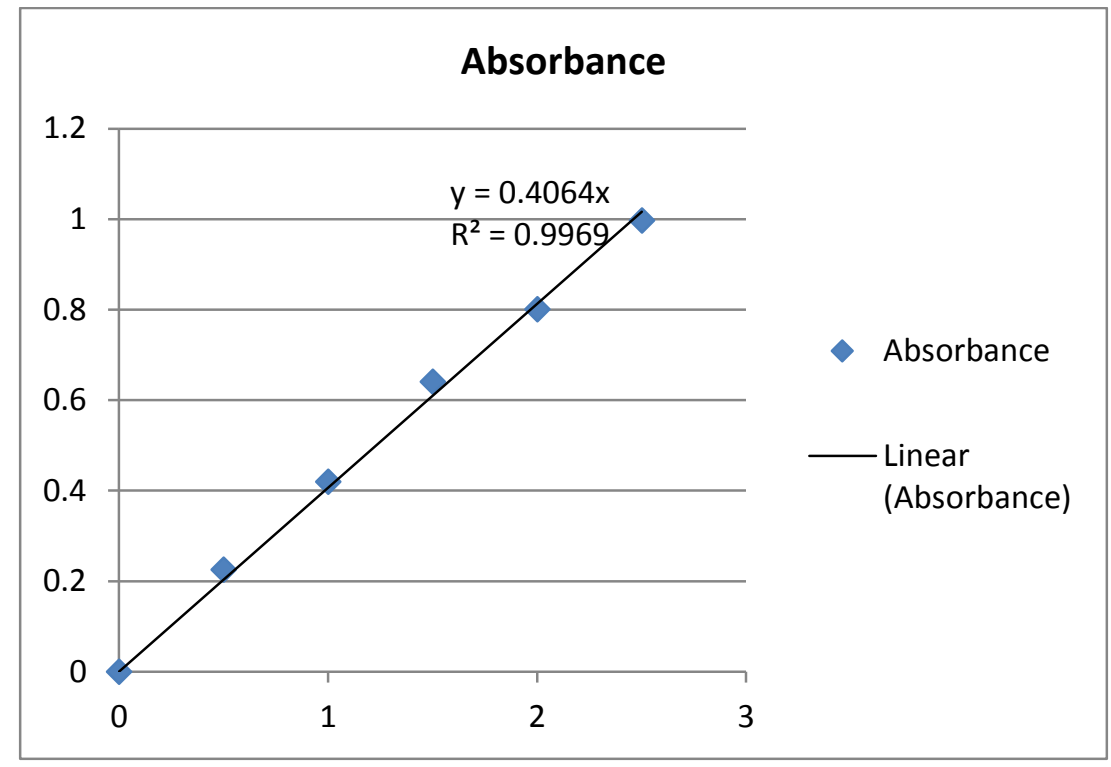

$\lambda$ max of drug in phosphate buffer $\left(P^{\mathrm{H}} \mathbf{7 . 4}\right)$

Standard calibration curve of drug in phosphate

\begin{tabular}{lll}
\hline S.No & Concentration & Absorbance \\
\hline 1 & 0.5 & 0.2251 \\
2 & 1 & 0.4195 \\
3 & 1.5 & 0.6407 \\
4 & 2 & 0.8013 \\
5 & 2.5 & 0.9971 \\
\hline
\end{tabular}

\section{Buffer (PH 7.4)}

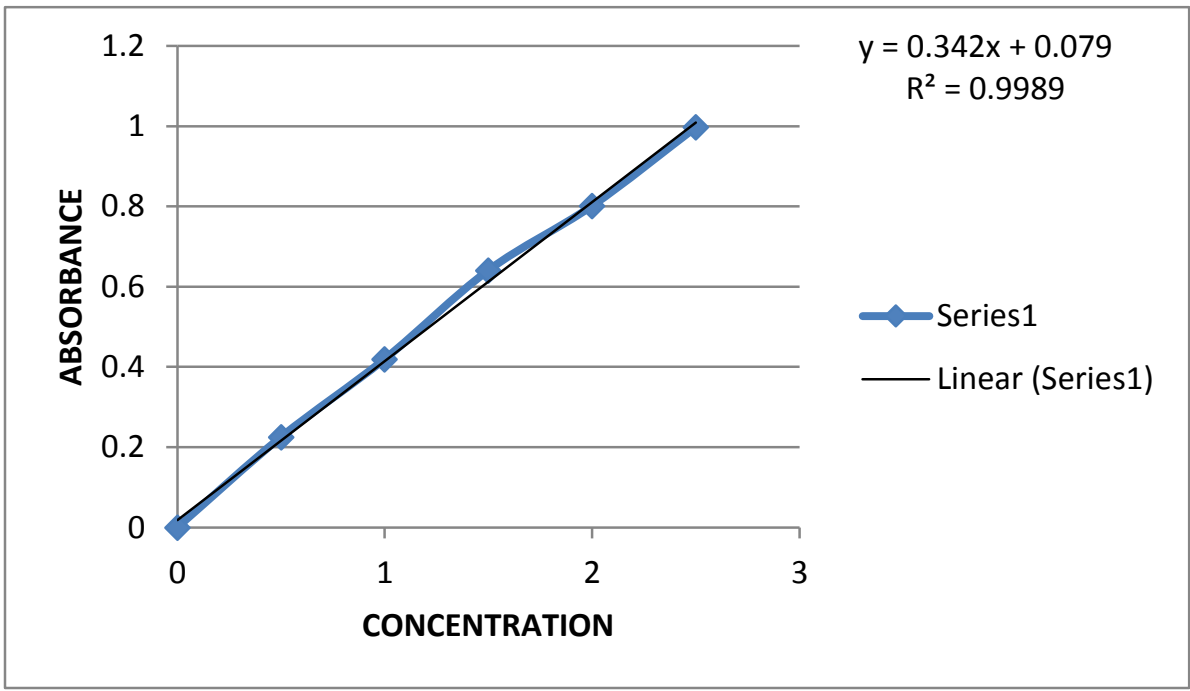

Standard curve of Ganciclovir in phosphate buffer (PH 7.4)

\section{Drug- excipient compatibility studies using FTIR:}

IR spectra's for pure drug as well as excipients used mannitol, phosphotidyl choline and cholesterol and also proliposomal formulation were recorded and compared to identify possible interaction. 


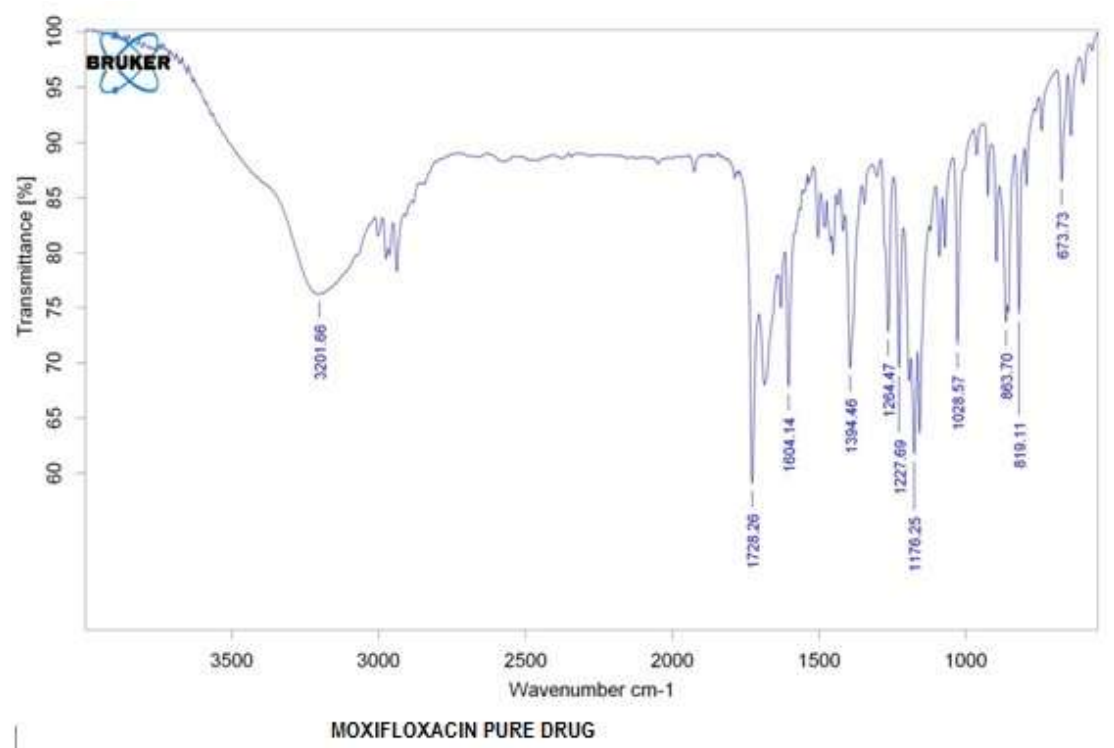

FTIR spectra of Ganciclovir pure drug

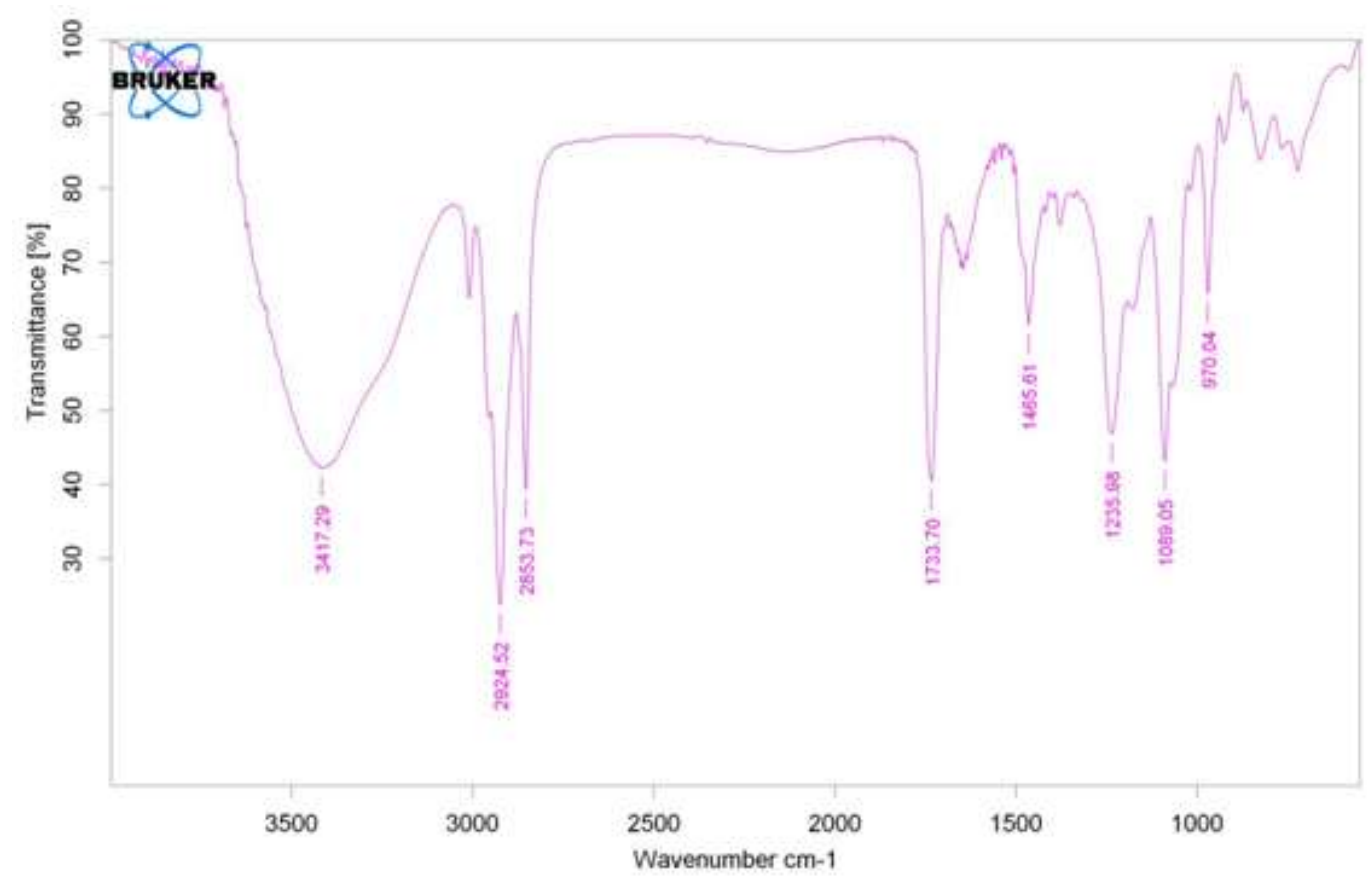

PHOSPHOTIDYL CHOLINE

FTIR spectra of Phosphotidyl choline

\begin{tabular}{|c|c|c|c|c|}
\hline \multirow[t]{2}{*}{ S.NO } & \multicolumn{2}{|c|}{$\begin{array}{l}\text { Wave number in } \\
\text { formulation }\left(\mathrm{cm}^{-1}\right)\end{array}$} & \multirow[t]{2}{*}{$\begin{array}{l}\text { Characteristic Wave } \\
\text { number range }\left(\mathrm{cm}^{-1}\right)\end{array}$} & \multirow[t]{2}{*}{$\begin{array}{l}\text { Bond nature and bond } \\
\text { attributed }\end{array}$} \\
\hline & Pure drug & $\begin{array}{l}\text { Optimized } \\
\text { formulation }\end{array}$ & & \\
\hline 1 & 3201 & 3404 & $3300-2500$ & O-H stretching Carboxylic acids \\
\hline 2 & 1720 & 1753 & $1760-1690$ & $\mathrm{C}=\mathrm{O}$ stretching Carboxylic acids \\
\hline 3 & 1600 & 1422 & $1600-1400$ & $\mathrm{C}-\mathrm{C}$ stretch in ring aromatics \\
\hline
\end{tabular}




\begin{tabular}{lllll}
\hline 4 & 1264 & 1282 & $1320-1000$ & C-O stretch Esters \\
5 & 883 & 880 & $900-675$ & C-H oop aromatics \\
6 & 673 & 680 & $1000-650$ & $=$ C-H bend Alkenes \\
\hline
\end{tabular}

\section{FTIR data interpretation}

On comparison of IR spectra of proliposomes, pure Ganciclovir drug, mannitol, cholesterol and phospholipid it was clear that, there was no significant interaction of encapsulated drug with the phospholipid and water soluble solid support (mannitol) with formulations

\section{Drug content of proliposomal formulations}

\begin{tabular}{lll}
\hline S.No & Formulation & \%Drug content \pm SD \\
\hline 1 & F1 & $95.03 \pm 0.543$ \\
2 & F2 & $86.4 \pm 0.734$ \\
3 & F3 & $93.7 \pm 0.664$ \\
4 & F4 & $96.8 \pm 0.249$ \\
5 & F5 & $94.7 \pm 0.984$ \\
6 & F6 & $94.8 \pm 0.860$ \\
7 & F7 & $92.4 \pm 1.70$ \\
8 & F8 & $90.6 \pm 0.748$ \\
9 & F9 & $87.5 \pm 0.953$ \\
\hline
\end{tabular}

The Ganciclovir content in the proliposomes were observed in the range of $86.4 \%$ to $96.8 \%$ at various drug to phospholipid ratios. From the above results it is concluded that F4, F1, F5 and F6 formulations showed maximum drug content when compare to other formulations

\section{In -vitro studies:}

The cumulative amount of drug release of various proliposomal formulations and conventional (Conventional) Ganciclovir gel after 16hrs were shown in table

Cumulative percentage drug release of proliposomal formulations

\begin{tabular}{llllllllll}
\hline Time(MIN) & \% Drug Release & & & & & & \\
& F1 & F2 & F3 & F4 & F5 & F6 & F7 & F8 & F9 \\
\hline 0 & 0 & 0 & 0 & 0 & 0 & 0 & 0 & 0 & 0 \\
5 & 24.8 & 21.5 & 19.6 & 23.6 & 14.4 & 17.22 & 18.22 & 20.7 & 21.6 \\
10 & 30.61 & 27.31 & 25.66 & 27.22 & 23.8 & 18.04 & 22.56 & 23.92 & 23.98 \\
15 & 36.43 & 33.12 & 32.12 & 31.87 & 27.45 & 24.15 & 26.78 & 27.45 & 28.45 \\
20 & 40.2 & 37.48 & 38.02 & 36.87 & 33.45 & 28.6 & 29.45 & 30.78 & 33.45 \\
25 & 50.98 & 44.23 & 41.67 & 40.67 & 41.77 & 39.87 & 35.71 & 36.67 & 38.95 \\
30 & 69.65 & 48.13 & 48.15 & 60.22 & 50.98 & 45.56 & 34.87 & 47.89 & 49.07 \\
35 & 71.22 & 49.55 & 51.06 & 67.89 & 60.61 & 58.15 & 41.12 & 53.08 & 54.02 \\
40 & 75.66 & 50.38 & 53.07 & 78.2 & 64.77 & 60.79 & 52.06 & 59.1 & 69.81 \\
45 & 76.01 & 55.4 & 55.44 & 80.55 & 69.88 & 64.5 & 58.94 & 60.05 & 74.1 \\
\hline
\end{tabular}




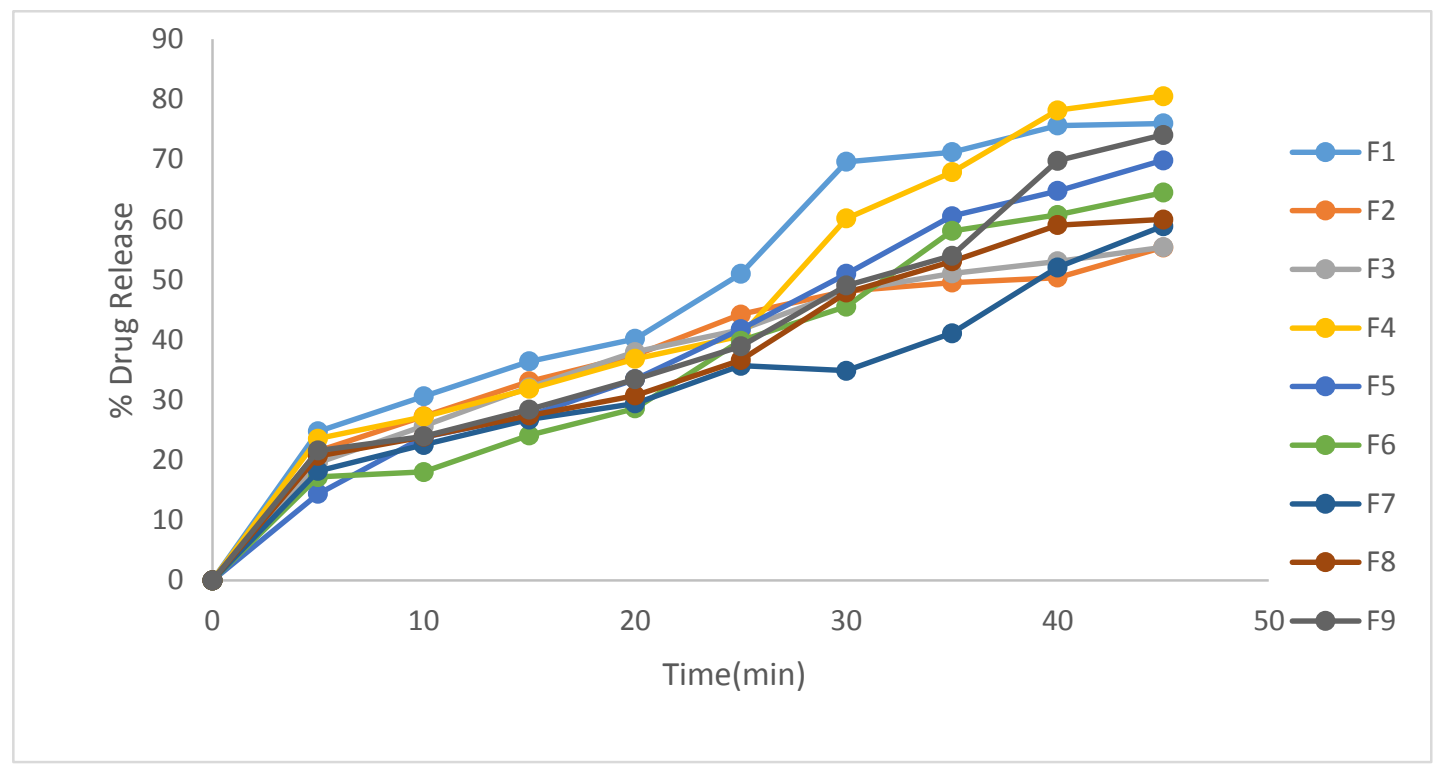

Diffusion data of various proliposomal formulations and conventional

From the above results $\mathrm{f} 4$ formulation has selected as optimized one which is used for further study.

Particle size count and distribution:
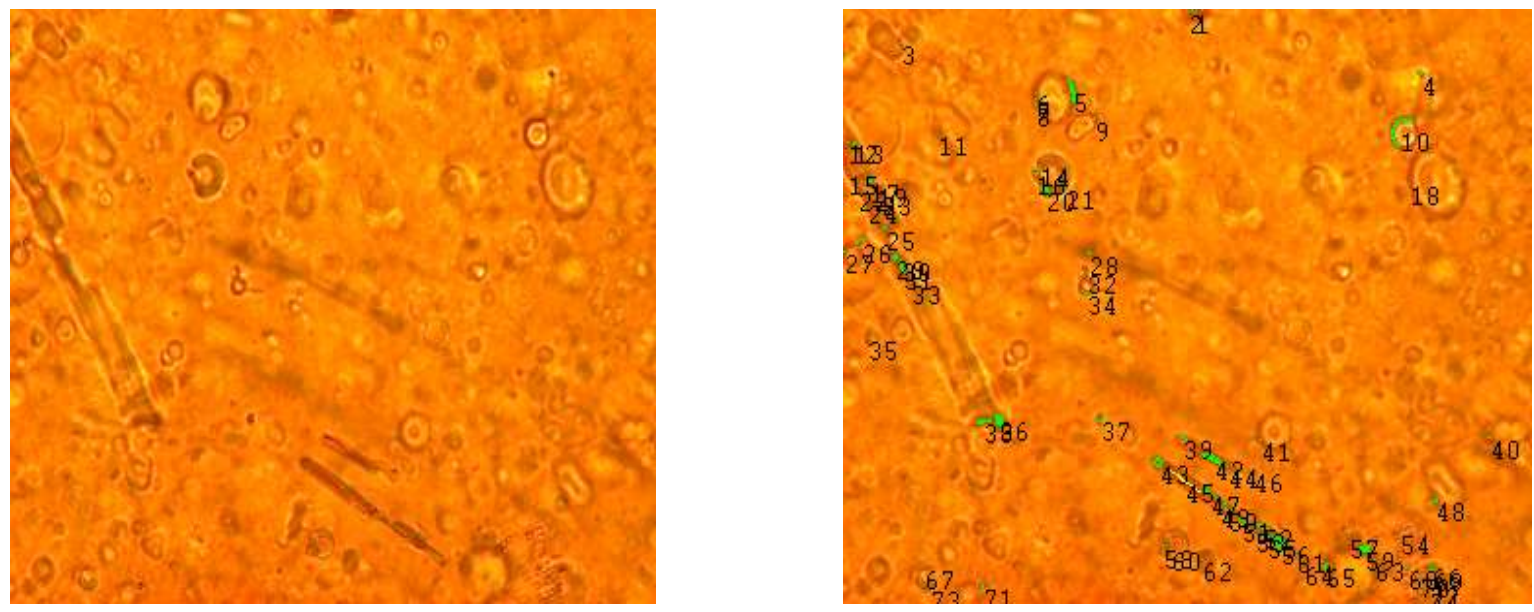

Particle size distribution of optimized formulation

\section{Determination of zeta potential}

Zeta potential was measured by using zeta sizer ( Melvern, INDIA) and was found to be $-16 \mathrm{mv}$ which is sufficient to avoid aggregation of vesicles. From the reports of zeta sizer vesicle size was found to be $990 \mathrm{~nm}$.

Zeta size distribution by intensity

\begin{tabular}{lllll}
\hline Results & Peaks & Diameter(nm) & \% Intensity & Width (nm) \\
\hline Z-average(d. nm) -990 & Peak 1 & 91.50 & 93.3 & 13.07 \\
PDI-0.82 & Peak 2 & 13.91 & 6.7 & 1.404 \\
Intercept-0.432 & Peak 3 & 0.000 & 0.0 & 0.00 \\
\hline
\end{tabular}




\section{Surface morphology:}

The surface morphology of proliposome granules, pure drug and mannitol granules was examined by scanning electron microscopy (SEM) and the images were photographed at 100 resolution.

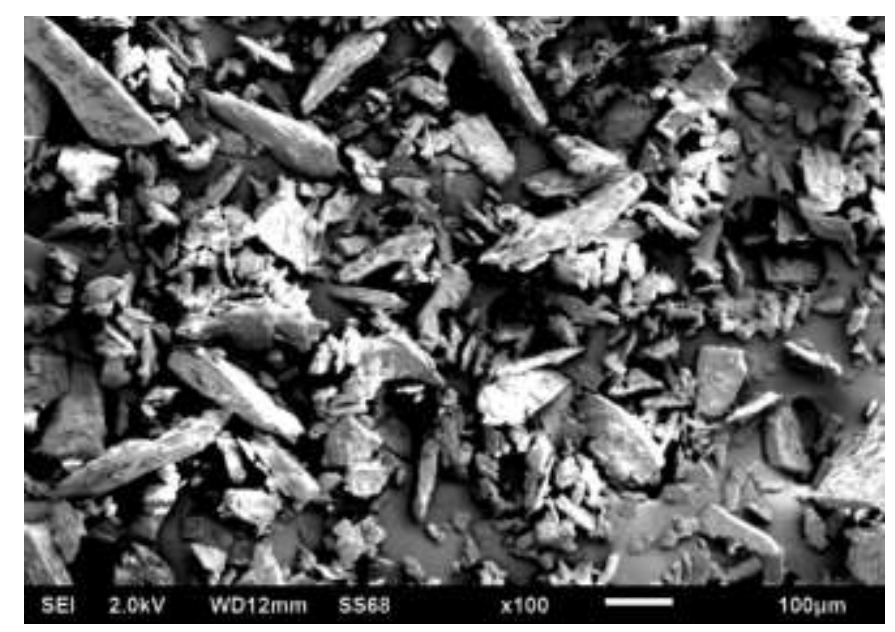

SEM image of Ganciclovir

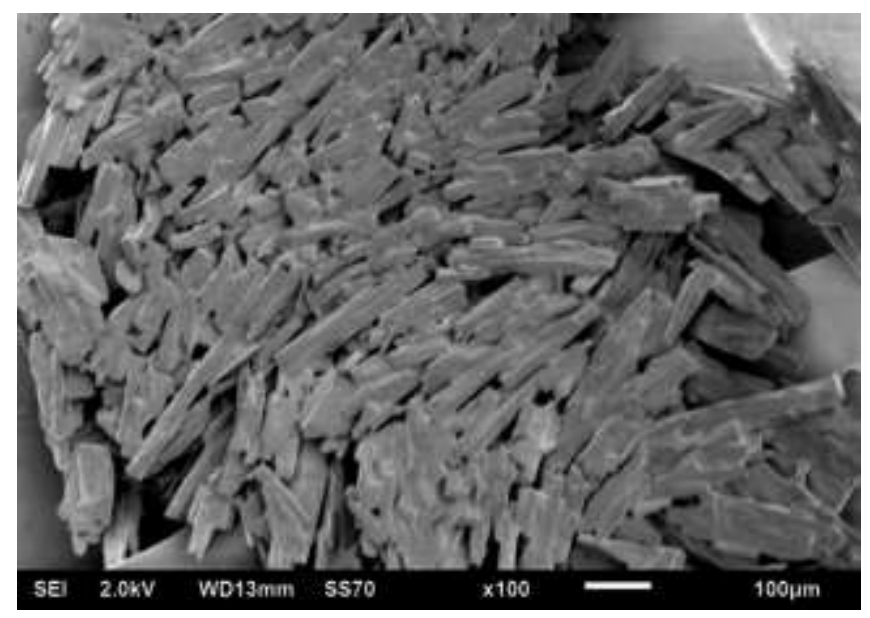

SEM image of Ganciclovir proliposomes

The surface morphology of proliposome granules and plain mannitol granules were examined by scanning electron microscopy. The surface morphology of proliposome powder was different as compare to plain mannitol powder as shown in SEM. From SEM photographs it is clear that, the surface of mannitol crystals becomes illegible due to deposition of phospholipid on mannitol surface.

\section{REFERENCES:}

1. Roaa A.Nief et al.,(2019)to design a mucoadhesive pH-triggered in situ gel of itraconazole ,to prolong the residence time on oral mucosa , and to reduce the frequency of application and amount of drug administered . Six formulations containing 
itraconazole were prepared different concentrations of carbopol934 and in combination with different viscosity-enhancing agents such as hydroxypropyl methylcellulose (HPMC), xyloglucan and hyaluronic acid to formulate a pH-triggered system that undergoes sol-to-gel transition on change in $\mathrm{pH}$ to the physiological $\mathrm{pH}$. The formulations were evaluated for physical appearance, $\mathrm{pH}$, gelation capacity, rheological properties , spreadability ,muco adhesive force, and in vitro release of drug

2. Dibyalochan mohanty et al.,(2018) the gel system has emerged as one of the best novel drug delivery system ; it helps for the sustained and controlled release of the drugs by its special characteristic features of sol to Gel transition. In situ gelling system is a formulation that is in solution form before entering into the body, but it will change to gel form under various physiological conditions

3. Mallesh Kurakula et al., "Formulation and Evaluation of Prednisolone Proliposomal Gel for Effective Topical Pharmacotherapy". International Journal of Pharmaceutical Sciences and Drug Research., 4(1).2012. Pp.35-43.

4. Pakhuri Mehta et al., "Development \& Validation of Stability-Indicating UV Spectrophotometric Method for Estimation of Naproxen in Pharmaceutical Dosage Form". Asian Journal of Biochemical and Pharmaceutical Research., 2 (1) 2012. pp. 2231-2560

5. Abid Hussain et al., "Effect Of Olive Oil on Transdermal Penetration of Flurbiprofen from Topical Gel as Enhancer”. Pak. J. Pharm. Sci., 25(2). 2012. pp.365-369

6. Vishal D.Yadav et al., "Effect of Carbopol 934 and 940 on Fluconazole Release from Topical Gel”. Current Pharma Research. 2 (2). 2012. pp. 487-493.

7. Divyesh patel et al., "Transdermal Drug Delivery System: Review". International Journal Of Biopharmaceutical \& Toxicological Research.,1 (1).2011. Pp.61-80

8. Mona hassan aburahma, Ghada ahmed abdelbary. "Novel Diphenyl Dimethyl Dicarboxylate Provesicular Powders With Enhanced Hepatocurative Activity: Preparation, Optimization, In Vitro/ InVivo Evaluation". International Journal Of Pharmaceutic.2011.

9. Wipaporn rojanarat et al., "Isoniazid Proliposome Powders For Inhalation- Preparation, Characterization And Cell Culture Studies”. Int. J. Mol. Sci., 12.2011.pp. 4414-4434.

10. Janga y. karthik et al., "Bioavailability Enhancement of Zaleplon Via Proliposomes: Role of Surface Charge". European Journal Of Pharmaceutics And Biopharmaceutics.2011. 
11. Ashok kumar et al., "Transdermal Drug Delivery System: An Overview”. International Journal of Pharmaceutical Sciences Review and Research.,3 (2).2010.pp.49-54.

12. Suvakanta dash et al., "Kinetic Modeling on Drug Release from Controlled Drug Delivery Systems”. Acta Poloniae Pharmaceutical - Drug Research., 67 (3).2010. pp. 217-223.

13. Dinesh VK, Suryaprakash TN, Eswari TS. Formulation, characterization and in-vitro evaluation of acarbose loaded ultra-deformable liposome gel for effective transdermal delivery. Adv Pharmacol Toxicol 2010;11(1):43-46.

14. S dhawan et al., "Formulation and Evaluation of Diltiazem Hydrochloride Gels for the Treatment of Anal Fissures". Sci Pharm. 77. 2009. Pp.465-482.

15. Dalia A. Attia et al., "In Vitro and in Vivo Evaluation of Transdermal Absorption of Naproxen Sodium". Australian Journal of Basic and Applied Sciences., 3(3). 2009. pp.2154-2165.

16. H. Xu et al., "Optimized preparation of vinpocetine proliposomes by a novel method and in vivo evaluation of its pharmacokinetics in New Zealand rabbits". J. Control. Release .140 .2009 . pp.61- 68

17. Vandana gupta et al., "Formulation Development and In Vitro Characterization of Proliposomes for Topical Delivery Of Aceclofenac". Indian Journal Of Pharmaceutical Sciences., 70(6).2008. pp.768-775.

18. Jing ming li et al.,(2008). "Preparation Of Stealthy Etoposide Proliposomes And The Pharmacokinetics In Rabbits”. Journal Of Chinese Pharmaceutical Sciences., 4.2008. pp.303-306.

19. Harris ED Jr, Firestein GS.. Textbook of Rheumatology - Clinical features of rheumatoid arthritis. 8th ed. Philadelphia, Pa: Saunders Elsevier. 2008.

20. Saag KG, Gim GT, Nivedita M et al., "American College of Rheumatology 2008 Recommendations for the Use of Nonbiologic and Biologic Disease-Modifying Anti rheumatic Drugs in Rheumatoid Arthritis". Arthritis \& Rheumatism. 59 (6).2008.pp.762784.

21. Xiao yan-yu et al.,(2006). "Preparation Of Silymarin Proliposome: A New Way to Increase Oral Bioavailability of Silymarin in Beagle Dogs”. International Journal Of Pharmaceutics., 319.2006. pp. 162-168.

22. Vladimir P. Torchilin. "Recent Advances with Liposomes as Pharmaceutical Carriers". Drug Discovery., 4. 2005.Pp.145-160 
23. Ansel, H. C., Popovich, N. G. and Allen, Loyd V.. Pharmaceutical Dosage Forms and Drug Delivery Systems. B. I. Publications Pvt. Ltd.2005.pp.415-419.

24. Mei-ying ning et al., "Preparation and Evaluation of Proliposomes Containing Clotrimazole”. Chem. Pharm. Bull., 53(6).2005. pp.620-624.

25. Langer R. "Transdermal Drug Delivery: Past Progress, Current Status and Future Prospects”. Adv Drug Deliv Rev., 56.2004.pp. 557-558.

\section{AJPHR is}

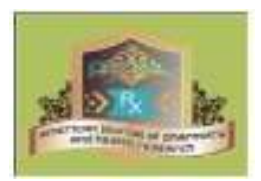

Peer-reviewed monthly

Rapid publication

Submit your next manuscript at

editor@ajphr.com / editor.ajphr@gmail.com 